\title{
Variability of Hubble's Parameter, Geomagnetic Activity, and Putative Changes in Space-Mass Density: Implications for Terrestrial Cell Growth
}

\author{
Michael A. Persinger \\ Laurentian University, Sudbury, Ontario, Canada P3E 2C6 \\ E-mail address: mpersinger@laurentian.ca
}

\begin{abstract}
Keywords: Proton Expansion; Hubble Constant; Geomagnetic Correlation; Background Photon Flux Density; Friedmann's Equation
\end{abstract}

\begin{abstract}
The quotient for Planck's Length divided by the product of Hubble's parameter and twice the width of the Compton wave length for a proton has been considered a critical increment for the time required for a proton to expand one Planck's Length. The empirical time of $3.25 \mathrm{~ms}$, found in magnetic field effectiveness for multiple physical and biochemical reactions, requires a local Hubble constant $(\mathrm{H})$ of $58 \mathrm{~km} \cdot \mathrm{s}^{-1} \cdot \mathrm{MPar}^{-1}$ with a resulting mass density of 0.14 protons per cubic meter. This mass density multiplied by the cube of the galactic orbital velocity is within error measurement of the background photon flux density measured locally by photomultiplier units over the last four years. Regression analyses for the weak positive correlation between Huchra's annual fluctuations in $\mathrm{H}$ and global annual geomagnetic activity over the last 30 years indicated that every $1 \mathrm{nT}$ increase was associated with $0.44 \mathrm{~km} \cdot \mathrm{s}^{-1} \cdot \mathrm{MPar}^{-1}$ increase in $\mathrm{H}$. The required average density is equivalent to that of the rest mass of the electron. The results and quantitative solutions indicate that the measurement of $\mathrm{H}$ is affected by geomagnetic activity and that the time for a proton to expand 1 Planck's Length can vary over time. Unless earth-based reactions from exposures to pulsed or "quantum well" like magnetic fields that depend upon resonant precision with this value are adjusted appropriately their efficacy could vary significantly.
\end{abstract}

\section{INTRODUCTION}

Matter exits within space and may be a special condition of the structure of space. If space is expanding then the matter should expand proportionally. The time required for this expansion is potentially predictable if an indicator of spatial expansion and the length of the space occupied by the matter were related to the smallest increment of space. Persinger and Koren [1] assumed these three features are Hubble's parameter (in the order of $2.2 \cdot 10^{-18} \mathrm{~s}^{-1}$, assuming $67.8 \mathrm{~km} \cdot \mathrm{s}^{-1} \mathrm{MPar}^{-1}$ ), the Compton wavelength $\lambda_{\mathrm{Cp}}$ for a proton $\left(1.32 \cdot 10^{-15} \mathrm{~m}\right)$ and Planck's Length $\left(1.616 \cdot 10^{-35} \mathrm{~m}\right)$. The simple relationship is:

$$
\mathrm{t}=\mathrm{P}_{\mathrm{L}}\left(\mathrm{H} \cdot \lambda_{\mathrm{Cp}}\right)^{-1}
$$

Given the specific contemporary estimate of Hubble's parameter the resulting temporal increment for a proton to expand 2 wavelengths $\left(2.64 \cdot 10^{-15} \mathrm{~m}\right)$ or $\mathrm{L}_{\mathrm{p}}$ was about $3 \mathrm{~ms}$. The potential for local phenomena to be affected by or reflected in such large-space phenomena as Hubble's constant is consistent with Mach's principle that matter in any given space is affected by all of the matter within the universe [2]. His argument was based upon mechanisms involving intermediate stages of momentum. Contemporary concepts of "excess correlation" over non-traditional distances or forms of "entanglement" could be considered modern manifestations of that principle [3]. Experimental results and concomitant calculations support the potential to infer universal properties from very local and simple procedures [4].

Several experiments have indicated that computer-generated magnetic fields whose serial point durations were $3 \mathrm{~ms}$ influenced the dynamics within shifts in $\mathrm{pH}$ of spring water [5], calcium influxes, photon emissions [6], and cellular activity that primarily involve the proton or chemical changes that depend upon the movement of protons [7]. Koren et al [8] suggested that the $3 \mathrm{~ms}$ 
duration required for a proton to expand an additional Planck's Length could be applied to infer H locally (at ground level) as an alternative method to inferring this value from red shifts of distant objects.

The optimal time $(t)$ for the point duration of magnetic fields that produced the specific slowing of growth in a variety of different cancer cell lines but not normal cells has been shown to peak around $3 \mathrm{~ms}$ while point durations of $2 \mathrm{~ms}$ or less or $4 \mathrm{~ms}$ or more were not effective. The resting membranes of most cancer cell lines are hypopolarized and vacillate around the 26 to $27 \mathrm{mV}$ range which is the semi-constant potential difference based upon the gas constant, the cells' thermal level in absolute temperature and Faraday's number for charge density without reference to the disparity of ion concentrations between the inside and outside of the membrane [9]. The energy associated with this value is the threshold where thermal oscillations and random processes dominate the system. They are quantifications consistent with universal "dynamic pressure" [10].

If the optimal time as noted in equation (1) is determined by $\mathrm{H}$, assuming that both $\mathrm{P}_{\mathrm{L}}$ and $\mathrm{L}_{\mathrm{p}}$ are constant, then any process that alters $\mathrm{H}$ would affect the optimal value. Here calculations and data are presented that suggest that the functional average mass density of space within which the solar system is immersed as it moves around the galaxy might change and hence alter the responses of cells operating near the kT Nernst threshold. This would be potentially relevant for treating malignant cells.

\section{ALTERNATIVE DETERMINATION OF H FROM G AND MASS DENSITY}

$\mathrm{H}$ can also be expressed as:

$$
\mathrm{H}^{2}=1 / 3 \cdot(8 \pi \mathrm{G} \sigma)
$$

where $\mathrm{G}$ is the Newtonian Gravitational constant $\left(6.67 \cdot 10^{-11} \mathrm{~m}^{3} \cdot \mathrm{kg}^{-1} \cdot \mathrm{s}^{-2}\right)$ and $\sigma$ is the average density of matter. This means that either a change in $\mathrm{G}$ or $\sigma$ could affect $\mathrm{H}$ which would determine the optimal duration for expanding one Planck's Length (i.e., double the wavelength). Depending upon the assumptions of geometry the additional terms are $+\left(\mathrm{k}^{2} \cdot \mathrm{a}^{-2}\right)-\lambda \mathrm{c}^{2} / 3$, which is Friedmann's equation (where $\mathrm{k}$ is $+1,0$, or -1 depending upon the universe's shape, a is the scaling factor, $\lambda$ is the cosmological constant, and $\mathrm{c}$ is the velocity of light), could produce small changes in this value. If these terms are ignored the relationship becomes:

$$
\mathrm{t}^{2}=\left(\mathrm{P}_{\mathrm{L}}^{2}\right) \cdot\left[(1 / 3 \cdot 8 \pi \mathrm{G} \sigma) \cdot\left(\mathrm{L}_{\mathrm{p}}^{2}\right)\right]^{-1}
$$

If we assumed $G$ does not fluctuate substantially in annual time and $\sigma=1.67 \cdot 10^{-27} \mathrm{~kg} \cdot \mathrm{m}^{-3}(1$ proton per cubic meter) which would constitute Eddington's Number within a characteristic universal volume, the time $(t)$ required for a proton to expand one Planck's Length would be $\sim 20$ ms. This density would be equivalent to a Hubble's parameter of $3.05 \cdot 10^{-19} \mathrm{~s}^{-1}$ or a Hubble's constant of $9.39 \mathrm{~km} \cdot \mathrm{s}^{-1} \cdot \mathrm{MPar}^{-1}$. For the more typical contemporary average value for Hubble's constant of $70 \mathrm{~km} \cdot \mathrm{s}^{-1} \cdot \mathrm{MPar}^{-1}\left(2.26 \cdot 10^{-18} \mathrm{~s}^{-1}\right.$ ), $\sigma=9.14 \cdot 10^{-27} \mathrm{~kg} \cdot \mathrm{m}^{-3}$ (or about 5.47 proton mass equivalents). The resulting $\mathrm{t}=2.7 \mathrm{~ms}$.

\section{INTERRELATIONSHIPS TO OPTIMAL POINT DURATIONS THAT SLOW MALIGNANT CELLULAR GROWTH}

We have found in the last four years that the optimal duration for the point durations of applied physiologically-patterned (primarily temporally asymmetric frequency and phasemodulated) magnetic fields to produce the most significant disruption of proliferation of malignant cell growth without affecting normal cells is about $3.25 \mathrm{~ms}$. The band is relatively narrow, i.e., between 3.0 and $3.5 \mathrm{~ms}$. The primary mechanism involves proton movements associated with $\mathrm{pH}$ coupled to calcium influx particularly through T-type channels [11]. These small changes in the $\mathrm{pH}$ of the medium, particularly when the shift is from neutral (7.4) to mildly acidic (6.8), are associated 
with marked increases in photon emissions from the immersed cells as measured by digital photomultiplier units. This occurs when the magnetic field composed of successively created voltage values with the optimal point duration is applied. Simple cellular proliferation diminishes with this mildly acetic $\mathrm{pH}$. Experimental reduction to more acidic levels, before membrane dissolution occurs, produces no additional effect.

According to the above equations the optimal Hubble parameter for a proton to double its Compton wavelength in $3.25 \mathrm{~ms}$ would be $1.88 \cdot 10^{-18} \mathrm{~s}^{-1}$. This is equivalent, when multiplied by a MPar $\left(3.086 \cdot 10^{22} \mathrm{~m}\right)$, to $58 \mathrm{~km} \cdot \mathrm{s}^{-1} \cdot \mathrm{MPar}^{-1}$. This is below the contemporary estimates of around 70 $\mathrm{km} \cdot \mathrm{s}^{-1}$ but it has been measured at least once most years according to the collection of $\mathrm{H}$ measurements by John Huchra [12] from the Harvard-Smithsonian Center for Astrophysics.

The equivalent average mass density for this Hubble's constant $\left(58 \mathrm{~km} \cdot \mathrm{s}^{-1} \cdot \mathrm{Mpar}^{-1}\right)$ would be:

$$
\sigma=\left(\mathrm{H}^{2} \cdot 3\right) \cdot(8 \pi \mathrm{G})^{-1}
$$

The resulting value is $2.38 \cdot 10^{-28} \mathrm{~kg} \cdot \mathrm{m}^{-3}$ or about 0.14 particle per cubic meter. The quantity is within measurement error of the current estimates of 0.2 particles per cubic meter in some popular cosmological models. To obtain flux power density of photons from mass density the intermediate cube of velocity is required. The most reasonable velocity that would be expected to influence local values of $\mathrm{H}$ would be the orbital speed of the solar system around the galactic center. The value is assumed to be about $242 \mathrm{~km} \cdot \mathrm{s}^{-1}$. The product of the mass density (sufficient to produce the optimal Hubble parameter) and the cube of the velocity is $3.27 \cdot 10^{-12} \mathrm{~W} \cdot \mathrm{m}^{-2}$.

This is the same order of magnitude (and almost identical coefficient) of the background fluctuations in photon flux density we have been measuring in our laboratory in the Sudbury Basin once per minute for the last four years. Concurrent measurements of fluctuations in geomagnetic field intensity from a magnetometer sensor within $0.25 \mathrm{~m}$ of the sensor for the photomultiplier tube have shown weak but persistent negative correlations. Regression analysis indicated that for ever $10^{-12} \mathrm{~W} \cdot \mathrm{m}^{-2}$ increase in photon energy there was a concomitant decrease of $1 \mathrm{nT}$ in the earth's magnetic field intensity during quiet (no magnetic storms) conditions [13]. The range in the minute-to-minute fluctuation per day is $10^{-12}$ to $10^{-11} \mathrm{~W} \cdot \mathrm{m}^{-2}$. The primary factors that enhance the output over this background are the imminence of seismic events greater than magnitude 7.0 and particularly $M>8.0$ anywhere on the planet [14].

\section{POTENTIAL ANOMALIES: HUBBLE'S PARAMETER AND GLOBAL GEOMAGNETIC ACTIVITY}

The valuable list from Huchra's NASA-Smithsonian collection reveals the narrowing of the Hubble parameter over approximately four decades (Figure 1). From some perspectives one would not expect a property of space (reflected in by $\mathrm{H}$ ) to be affected by geomagnetic activity secondary to changes in solar activity. There are two demonstrations for the pursuit of this possibility. First, assuming a typical coronal mass ejection of $10^{12} \mathrm{~kg}$ into interplanetary space [15] and the distribution of this mass throughout the functional volume of the solar system, bounded by the heliopause (about $10^{39} \mathrm{~m}^{3}$ ), the resulting mass density would be $\sim 10^{-27} \mathrm{~kg} \cdot \mathrm{m}^{-3}$. This value is very proximal to the suspected average background of the mass density of the universe. If the process operates similar to other systems convergent or near-identical intensities are more likely to be resonant.

Secondly, calculations indicate that the energies associated with Gravity and photons may share a common source of variance [16]. There is the obvious solution for the duration of the universe (about 98 billion years) derived from the product of density $\left(1.67 \cdot 10^{-27} \mathrm{~kg} \mathrm{~m}^{-3}\right)$ and $\mathrm{G}$. Within the estimated volume of the universe this results in $\sim 10^{79}$ protons which are consistent with Eddington's Number and an equivalent mass of $10^{52} \mathrm{~kg}$. As $\lambda$ this value occurs as a component of the cosmological constant in order to accommodate Friedmann's equation. 
There is also the frequent inverse relationship between variations in $G$ measured on the earth's surface and geomagnetic activity [17]. A similar inverse correlation between local static geomagnetic field intensities of about 5 to $7 \mathrm{nT}$ and photon emissions have been shown for brain activity of human beings engaging in imagery [18] as well as for melanoma cells removed from incubation [19]. The range of intensity between 1 and $10 \mathrm{nT}$ may reflect the non-potential field which traditionally was employed to resolve inconsistencies for solid spherical harmonics when only internal and external sources geomagnetism were considered. Winch et al [20] showed that these non-potential fields can accommodate the anisotropy between gradient expressions for northwards and eastwards components of the earth's magnetic field as well as the corresponding radial current densities.

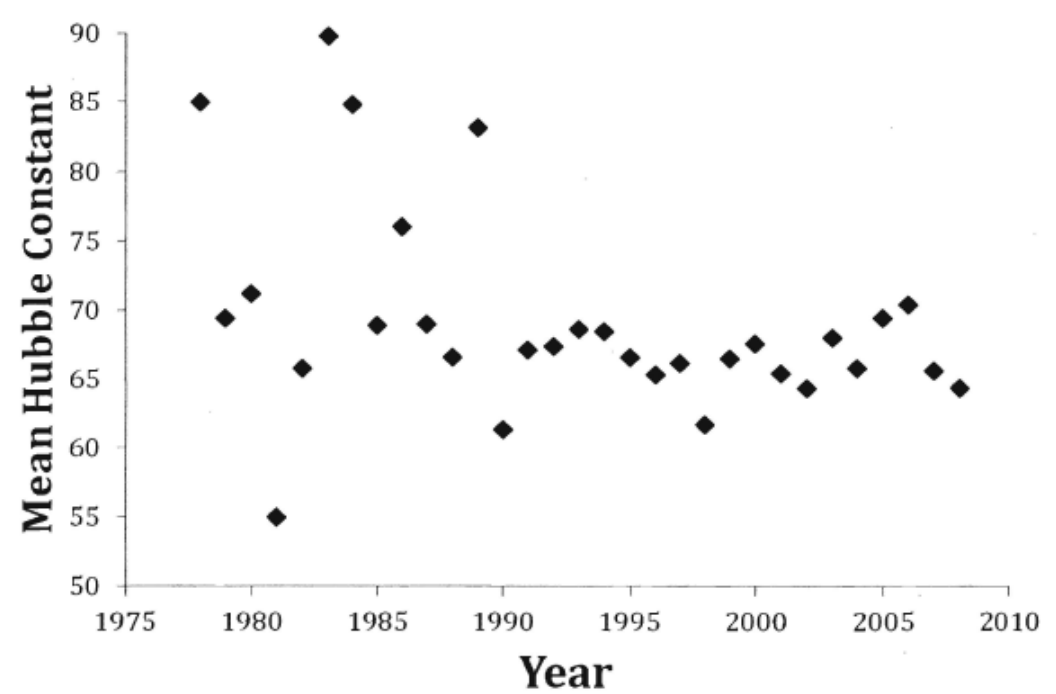

Figure 1. Mean value of Hubble's constant as a function of year of publication according to the Huchra data base

This discrepancy in E-W vs N-S components has been considered critical for demonstrating "excess correlation" or entanglement between spaces separated by non-traditional distances that share the same angular accelerating circular magnetic fields. This condition occurs with field strengths of about $5 \mathrm{nT}$ [21]. It may be relevant that the latency to respond to these "superpositioned" loci sharing the same circularly rotating pulsed $(1 \mathrm{~ms})$ magnetic fields is about 35 to 45 $\mathrm{s}$ which is the discrepancy between the energy from the upper limit of the rest mass of a proton of $\sim 2 \cdot 10^{-52} \mathrm{~kg}$ according to Tu et al [22] accelerated to $\mathrm{c}\left(\mathrm{m} \cdot \mathrm{c}^{2}\right)$ and Planck's constant. It may be relevant that the ratio of the rotational velocity of the earth at the latitude of experiments and $g(9.8$ $\mathrm{m} \cdot \mathrm{s}^{-2}$ ) is also within this range.

To discern if there were any associations between values for Hubble's constants and global geomagnetic intensity variations (as inferred by aa, or average antipodal, values) per year a series of correlations and partial correlations were completed for these values fr0m 1977 through 2009 . The Hubble constant values were obtained by taking the yearly mean and standard deviations for the measurements $(\mathrm{n}=531)$ from Huchra's NASA/HST Key Project (Extragalactic Distance Scale) and the average yearly aa values and sunspot (Wolf) numbers were obtained from M. A. El Borie [23]. The analysis began with 1977 because there were $\mathrm{H}$ values for every year thereafter until the end of Huchra's collection that was available to us. All analyses involved SPSS PC-16 software. For those 31 years the means and standard deviations (in parentheses) for these values were: mean $\mathrm{H}: 69.1$ (7.4), standard deviation of $\mathrm{H}: 14.1$ (5.0), coefficient of variation (SD divided by mean): $21 \%$ ( $8 \%$ ), aa values (in nT): 23.9 (5.5), and sunspot numbers: 72.7 (51.8). The latter was included to discern if the geomagnetic contribution shared the same source of variance as the fluctuations in sunspot numbers.

Because there were likely to have been delays between observation (measurement) and publication, 1 to 3 year lags were calculated for the aa values and sunspot numbers. To ensure temporal asymmetry, which would minimize the likely of drift artifact, 1 to 3 year lags were 
completed for the mean Hubble values as well. The correlations between yearly aa values and the four years of lag $(0,1,2,3)$ for Hubble means were $0.24,-0.24,-0.24$, and -0.01 , respectively and were not significant statistically $(\mathrm{p}>0.05)$. The correlation with sunspot numbers ranged from .05 to -0.07 (n.s.).

However the correlations (Pearson's r) between mean $\mathrm{H}$ and the four lags for aa values were $0.26,0.35,0.26$, and -0.26 . The first lag $(r=0.35)$ was significant at the $p<.06$ level. The nonparametric Spearman rho value was $0.44(\mathrm{p}=.01)$. The correlation between the mean annual $\mathrm{H}$ value and average annual geomagnetic variation is shown in Figure 2. The source of the unusual heteroschedasticity is unclear. The Pearson correlations between $\mathrm{H}$ values and sunspot numbers were $0.01,-0.12,0.05$, and .26 , respectively (n.s). Although there were no statistically significant associations between the coefficient of variation of $\mathrm{H}$ and aa values, the association with sunspot numbers and $\mathrm{H}$ occurred for these numbers for one year before $(\mathrm{r}=0.37)$ and two years before (0.38), the date of entry into Huchra's table.

To remove any occluded variance, partial correlations were completed. When the shared variance between geomagnetic activity and sunspot numbers was first removed, the partial correlations between $\mathrm{H}$ and the four lags were: $0.43(\mathrm{p}<0.05), 0.40(\mathrm{p}<0.05), 0.23$ and -0.11 . In other words when the effect of the sunspot numbers was removed the positive correlation between mean $\mathrm{H}$-independent geomagnetic activities became consistently statistically significant. When a similar analysis was completed for sunspot numbers after removal of the shared variance with aa activity, the $\mathrm{H}$ associations were still not significant statistically.

These results indicated the variance associated with geomagnetic activity independent of sunspot numbers was related to the $\mathrm{H}$ variability. To further discern if the aa values rather than sunspot numbers shared a source of variance with mean Hubble's constants per year factor analyses were completed for the Hubble values, the aa values for the same year and the previous year, and the sunspot numbers for the same year and previous year. After varimax rotation the loadings were obvious. Because of the small sample size the criteria set for statistical significance was $r>0.50$ rather than the traditional $>0.40$.

The first factor (loading coefficients in parentheses) was dominated by aa (0.66), sunspot numbers $(0.91)$ and sunspot numbers the year previously $(0.95)$. The second factor was loaded exclusively the Hubble values (0.83), aa (0.53) and aa values the previous year (0.77) only. These results also strongly suggest that the variations in yearly Hubble values were coupled to some source of variance which it shared with geomagnetic activity independent of solar activity. To check if the association was confounded by a serial correlation, that is, if the relationship was spuriously coupled to temporal progression (years), year was added to the variables for factor analyses. The factor structure containing the Hubble values did not change significantly.

To obtain the quantitative relationship between the $H$ value and aa value, regression analyses were completed. The slope was 0.44 which indicated that for every 1 unit increase in aa value $(1 \mathrm{nT})$ there was an increase of $0.44 \mathrm{~km} \cdot \mathrm{s}^{-1} \cdot \mathrm{Mpar}^{-1}$ in the measured Hubble constant. This means that over the range of the aa values ( 15 to $35 \mathrm{nT}$ ) there would be a change (increase) of 15 $\mathrm{km} \cdot \mathrm{s}^{-1} \cdot \mathrm{MPar}^{-1}$ in Hubble's value. Considering the standard error of the estimate $(0.23)$ of the equation this estimate would be not exact. 


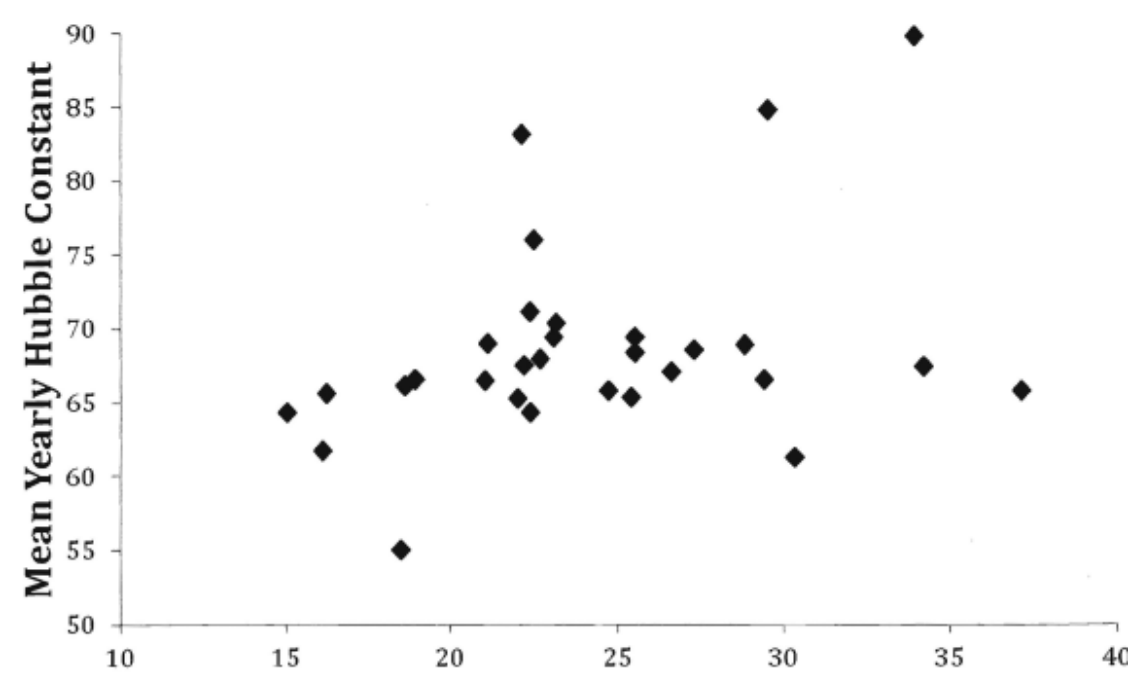

Average Yearly Global Geomagetic Activity

Figure 2. The annual average of Hubble's constant a function of the annual global geomagnetic activity the previous year. It is assumed that the one year lag reflects latencies for publication of results rather than an actual time constant for a physical effect.

The mass density from equation (4) for an increase in $\mathrm{H}$ of $0.44 \mathrm{~km} \cdot \mathrm{s}^{-1} \cdot \mathrm{Mpar}^{-1}$ would be $\sim 3.78 \cdot 10^{-31} \mathrm{~kg}$. In other words the increase in that velocity of $\mathrm{H}$ would be associated with an increase in mass density that could approach within $2 \pi$ that of electron at rest. The inference that perturbations in average mass density are compensated by local Hubble constant perturbations was developed by Zeldovich [24] over 30 years ago. If the coupled increase in geomagnetic activity of 1 nT is veridical then this specific increase in magnetic flux density in order to compliment the putative proton density should be related to some essential structural or spatial property of the electron. One possibility is the electric dipole moment of a unit electron which has been recently measured at $\sim 8.7 \cdot 10^{-31} \mathrm{~A} \cdot \mathrm{s} \cdot \mathrm{m}$ [25]. The product of this value and $10^{-9} \mathrm{~T}\left(\mathrm{~kg} \cdot \mathrm{A}^{-1} \cdot \mathrm{s}^{-2}\right)$ is $8.7 \cdot 10^{-40}$ $\mathrm{kg} \cdot \mathrm{m} \cdot \mathrm{s}^{-1}$.

Such a momentum term would be predicted by Mach to be an in integral part of the principle that each part of the universe is affected by every other part. We [26] have shown that an "entanglement velocity" derived from a diffusivity term emerges from two separate approaches when the entire universal set is involved $\left(\mathrm{G}^{2}, \mathrm{~kg}^{2}, \mathrm{~m}\right.$ and $\mathrm{t}^{3}$ terms, referring to Newton's Gravitational constant, the universe's mass, its diameter and its duration, respectively) is $2.8 \cdot 10^{23}$ $\mathrm{m} \cdot \mathrm{s}^{-1}$. The value for momentum when multiplied by entanglement velocity is a specific quantity of energy: $2.4 \cdot 10^{-16} \mathrm{~J}$. This is equivalent to $3.68 \cdot 10^{17} \mathrm{~Hz}$. When divided into the velocity of light in a vacuum the wavelength is $8.1 \cdot 10^{-10} \mathrm{~m}$. Considering the variability in the estimates for the contributing variables, the proximity of this distance to the circumference of the Bohr magneton may not be incidental. If this is valid, then the magnetic field strength of $1 \mathrm{nT}$ associated with the diminishment of $\mathrm{H}$ which is primarily proton-based would be reflected in the spatial extent of the circumferential distribution of the other fundamental energy, the electron, around the proton.

This potential gravitational interface is also manifested when the magnetic moment of the electron is considered. The value $\left(9.29 \cdot 10^{-24} \mathrm{~J} \cdot \mathrm{T}^{-1}\right)$ multiplied by $1 \mathrm{nT}$ results in an increment of energy of $9.29 \cdot 10^{-33} \mathrm{~J}$. The frequency equivalent, when divided by Planck's constant, is $14 \mathrm{~Hz}$. The exactness of this specific frequency is relevant in light of the innovative mathematical solutions by Minakov et al [27] who pursued the gravitational-to-electromagnetic wave conversion within electrostatic fields such as the earth-ionosphere resonator. Their primary solution for a plane gravitational wave interacting with a static electric field inside of the natural resonator formed between earth and the ionosphere was an EMF frequency response to the gravitational wave. The greatest power amplification was the second harmonic of the global Schumann Resonance, i.e., 14 Hz. Given the role of $\mathrm{G}$ in the solution for Hubble's parameter and the presence of intrinsic perturbations within this "constant" which is within the $10^{-14} \mathrm{~m}^{3} \cdot \mathrm{kg}^{-1} \cdot \mathrm{s}^{-2}$ range [28,29], the 
conditions could be created to produce such "interfaces" within the earth-ionosphere cavity as the solar systems moves through different densities of primary space during its orbit around the galaxy.

\section{POTENTIAL ANOAMLIES: ESCALATING PHOTON FLUX VALUES FROM INCREASED LOCAL MATTER DENSITY}

During the last three years we have noted a gradual increase in the background PMT measurements, not related to imminent earthquake events of $M>7.0$. Although there is a low probability it is related to instrument artifact, the tube has not failed and there are no technical indications of dysfunction. The average daily values are now more than 50 units higher than the baseline when the experiments began more than four years ago. The major inflection began around June, 2014 and escalated again in March 2015.

Assuming our empirically derived value of $5 \cdot 10^{-11} \mathrm{~W} \cdot \mathrm{m}^{-2}$ per unit for the photomultiplier meter ( 1 to 100 , standardized at 50 units), an increase of 50 would be equivalent to $250 \cdot 10^{-11} \mathrm{Wm}^{-2}$. Divided by the cube of the galactic orbital velocity of the solar system $\left(14.17 \cdot 10^{15} \mathrm{~m}^{3} \cdot \mathrm{s}^{-3}\right)$, the equivalent mass density would be $1.76 \cdot 10^{-25} \mathrm{~kg} \cdot \mathrm{m}^{-3}$. Although this is $\sim 105$ times the mass of a unit proton, it is much less than the $10^{6}$ protons per cubic meter contained within the interplanetary magnetic field. It is more likely to be observed as phase-shifts at the lower end of the frequency band in spectral power densities for these time-dependent variations. If this density is real, it could be sufficient to affect the parameters of space within which the expansion time of protons occur. The equivalent "dynamic pressure" of this mass density would be (after multiplying the velocity of light in a vacuum) $1.59 \cdot 10^{-8} \mathrm{~Pa}$.

For cells this would be very relevant if the intrinsic energies matched those of elementary particles. For melanoma cells with a diameter of $10 \mu \mathrm{m}$ and a volume of $5.26 \cdot 10^{-16} \mathrm{~m}^{3}$ the intrinsic energy from this elevated dynamic pressure would be $8.35 \cdot 10^{-24} \mathrm{~J}$. This is a recognizable value for physical scientists. When divided by the square of the velocity of light, it is the mass equivalent of $9.27 \cdot 10^{-31} \mathrm{~kg}$. This is remarkably proximal to the classic rest mass of an electron: $9.11 \cdot 10^{-31} \mathrm{~kg}$. Assuming this is not coincidence, the suggestion is that when the background photon flux density from moving around the galaxy achieves this value the activity of electrons within the cell could be minutely affected and subsequently be prone to resonance interaction.

The optimal point duration for this to occur can be derived from equation (3) while still considering the proton. With this specific mass density the optimal duration for the proton to expand two wavelengths would be almost exactly $1 \mathrm{~ms}$. The $1 \mathrm{~ms}$ duration is the intrinsic value shown for the electron to expand one classic diameter by both calculation and inferred experiments with excess correlation involving photon emissions. Given measurement error the actual value would be around $0.5 \mathrm{~ms}$.

\section{CONCLUSIONS}

If matter is a special condition or property of space than expansion of space is associated with expansion of matter when manifested as particles. The theoretical calculations and provisional empirical results indicated that the time for the expansion of a proton to twice its Compton wavelength could vary because of time-dependent changes in Hubble's constant. In a neutral Friedmann universe where $\mathrm{H}$ is dependent upon $\mathrm{G}$ and mass density only, the variations in the latter within values considered and inferred from measurements would be sufficient to significantly alter the time for proton expansion and hence the efficacy of point duration magnetic fields that optimally affect malignant cells. There is correlative evidence that local $\mathrm{H}$ may be quite different from distal estimates and that annual variations in $\mathrm{H}$ are coupled to a source of variance shared with global geomagnetic activity independent of the solar component.

\section{ACKNOWLEDGEMENTS}

Thanks to Professor David A. E. Vares for obtaining the Hubble data base and Dr. B. T. Dotta for technical contributions. 


\section{References}

[1] M. A. Persinger, S. A. Koren. International Journal of Neuroscience 117 (2007) 157-175.

[2] J. Singh, Great Ideas and Theories of Modern Cosmology (1961) Dover, N.Y.

[3] A. D. Aczel, Entanglement: The Greatest Mystery in Physics (2002) Raincoast, Vancouver.

[4] M. A. Persinger, International Letters of Chemistry, Physics and Astronomy 11 (2013) 86-92.

[5] B. T. Dotta, N. J. Murugan, L, M. Karbowski, M. A. Persinger, International Journal of Physical Sciences 8 (2013) 1783-1787.

[6] B. T. Dotta, M. A. Persinger, Journal of Biophysical Chemistry 3 (2012) 72-80.

[7] T. E. DeCoursey, Physiological Reviews 83 (2002) 475-579.

[8] S. A. Koren, B. T. Dotta, M. A. Persinger, The Open Astronomy Journal 7 (2014) 1-6.

[9] M. A. Persinger, R. M. Lafrenie, International Letters of Chemistry, Physics and Astronomy 17 (2014) 67-77.

[10] M. A. Persinger, Journal of Physics, Astrophysics and Physical Cosmology 3 (2009) 1-3.

[11] C. A. Buckner, A. L. Buckner, S. A. Koren, M. A. Persinger, R. M. Lafrenie, PLOS One DOI: 10.1371 (2015).

[12] J. Huchra, Hubble Estimates, Harvard-Smithsonian Center for Astrophysics, huchra@cfa.harvard.edu.

[13] D. A. E. Vares, M. A. Persinger, Journal of Non-Locality 2-Nr.2 (2013) http://journals.sfu.ca/nonlocality/inded.php/nonlocality/article/view/41/39

[14] M. A. Persinger, G. F. Lafreniere, B. T. Dotta, International Journal of Geosciences 3 (2012) 627-629.

[15] J. T. Gosling, Physics Fluids B 5 (1993) 2638-2645.

[16] M. A. Persinger, The Open Astronomy Journal 5 (2012) 41-43.

[17] M. A. Persinger, L. S. St-Pierre, International Journal of Geosciences 5 (2014) 450-452.

[18] M. A. Persinger, B. T. Dotta, K. S. Saroka, M. A. Scott, Journal of Consciousness \& Research 4 (2013) 1-24.

[19] M. A. Persinger, B. T. Dotta, L. M. Karbowski, N. J. Murugan, FEBS Open Bio 5 (2015) 413418.

[20] D. E. Winch, D. J. Ivers, J. P. R. Turner, R. J. Stening, Geophysical Journal International, 160 (2005) 487-504.

[21] N. Rouleau, M. A. Persinger, Journal of Electromagnetic Analysis and Applications 7 (2015) 19-30.

[22] L-C. Tu, J. Luo, G. T.Gilles, Reports on Progress in Physics 68 (2005) 77-130.

[23] M. A. El-Borie, S. S. Al-Thoyaib, International Journal of Physical Sciences 1 (2006) 67-74.

[24] Ya. B. Zeldovich, Cosmological fluctuations produced near a singularity. Monthly Notices of the Royal Astronomical Society 192 (1980) 663-667.

[25] J. Baron, W. C. Campbell, D. DeMille, J. M. Doyle, G. Gabrielse, Y. V. Gurevich, P. W. Hess, et al, Science 343 (2014) 269-272.

[26] M. A. Persinger, S. A. Koren, International Letters of Chemistry, Physics and Astronomy 3 (2015) 106-112. 
[27] A. A. Minakov, A. P. Nikoleanko, L. M. Rabinovich, Radiofiziks 35 (1992) 488-497.

[28] B. M. Vladimirskii, Biophysics 40 (1995) 915-923.

[29] T. Quinn, H. Parks, C. Speake, R. Davis, Physical Review Letters 111 (2013) 101102(5). 